\title{
CNAT Probe Normalization Method
}

National Cancer Institute

\section{Source}

National Cancer Institute. CNAT Probe Normalization Method. NCI Thesaurus. Code C81021.

A method for performing probe-level normalization of signal intensity to generate summaries file using CNAT method. 